\title{
4H-Silicon Carbide Wafer Surface after Chlorine Trifluoride Gas Etching Shogo Okuyama ${ }^{1}$, Keisuke Kurashima ${ }^{1}$, Ken Nakagomi ${ }^{1}$, Hitoshi Habuka ${ }^{1, a^{*}}$, Yoshinao Takahashi ${ }^{2, b}$ and Tomohisa Kato ${ }^{3, c}$ \\ ${ }^{1}$ Yokohama National University, 79-5 Tokiwadai Hodogaya Yokohama 240-8501 Japan \\ ${ }^{2}$ Kanto Denka Kogyo Co., Ltd, 2-105 Kanda Awaji-cho Chiyoda Tokyo 101-0063 Japan \\ ${ }^{3}$ National Institute of Advanced Industrial Science and Technology, 16-1 Onogawa Tsukuba 305-8569 Japan \\ ahabuka-hitoshi-ng@ynu.ac.jp, ${ }^{b}$ Yoshinao.Takahashi@kantodenka.co.jp ct-kato@aist.go.jp
}

Keywords: Silicon carbide dry etcher, chlorine trifluoride gas, etching rate profile.

\begin{abstract}
In order to develop the high etching rate reactor for silicon carbide, the $\mathrm{C}$-face $4 \mathrm{H}$-silicon carbide wafer, having the diameter of $50 \mathrm{~mm}$, was etched by the chlorine trifluoride gas at $500{ }^{\circ} \mathrm{C}$. By the deep etching, the concentric-circle-shaped valleys were observed at the positions corresponding to the radii of the pin-hole arrays of the gas distributor, as predicted by the calculation. The etching rate profile of $4 \mathrm{H}$-silicon carbide was concluded to have a relationship with the local chlorine trifluoride gas supply. The wafer distortion was small even very thin, about $160 \mu \mathrm{m}$ thick.
\end{abstract}

\section{Introduction}

Chlorine trifluoride $\left(\mathrm{ClF}_{3}\right)$ [1-6] gas is useful for quickly etching silicon carbide ( $\left.\mathrm{SiC}\right)$ material. In order to develop the wafer-scale etching process, the silicon carbide single-wafer dry etcher has been studied [3-6]. These previous studies using a polycrystalline 3C-silicon carbide wafer showed that the entire etching rate profile depended on the chlorine trifluoride gas concentration. The relationship between the etching rate distribution and the chlorine trifluoride gas concentration has been analyzed and clarified by means of the numerical calculations accounting for the transport phenomena $[4,5]$ in the reactor. Next, for applying this technique to the power device fabrication process, the $4 \mathrm{H}$-silicon carbide should be evaluated [7].

In this study, the etching capability of $\mathrm{C}$-face $4 \mathrm{H}$-silicon carbide single-crystalline wafer was studied using the chlorine trifluoride gas. Because the etched $\mathrm{C}$-face was specular, the entire etching behavior, such as the etching depth distribution and the wafer distortion, was studied by the visual inspection.

\section{Experimental Procedure}

Fig. 1 shows the cross-sectional view of silicon carbide dry etcher. The chlorine trifluoride gas was supplied from the gas injector, and was introduced to the wafer through the gas distributor. The gas distributor has 3-mm-diameter pin-holes arranged at the center and along five concentric circles, as shown in Fig. 2. The gas distributor and chamber wall were made of stainless steel. The gas mixture of chlorine trifluoride and nitrogen etched the silicon carbide wafer, which rotated at $10 \mathrm{rpm}$. The chlorine trifluoride gas reacts with the silicon carbide, with producing silicon tetrafluoride $\left(\mathrm{SiF}_{4}\right)$, carbon tetrafluoride $\left(\mathrm{CF}_{4}\right)$ and chlorine $\left(\mathrm{Cl}_{2}\right)$, as follows:

$$
3 \mathrm{SiC}+8 \mathrm{ClF}_{3} \rightarrow 3 \mathrm{SiF}_{4} \uparrow+3 \mathrm{CF}_{4} \uparrow+\mathrm{Cl}_{2} \uparrow
$$

The substrate used in this study was the mirror-polished C-face single-crystalline $4 \mathrm{H}$-silicon carbide wafer, having a diameter of $50 \mathrm{~mm}$ and a thickness about $160 \mu \mathrm{m}$. The wafer thickness was about a half of the ordinary-used wafer, for studying the wafer distortion cause by the deep etching, such as the wafer thinning process. Additionally, the wafer distortion is sensitively produced corresponding to the thermal and mechanical stress. 
The susceptor was made of quartz glass having a hollow shape. For heating the substrate to $500{ }^{\circ} \mathrm{C}$, halogen lamps and a gold reflector were set inside of the susceptor. The flow rate of chlorine trifluoride gas and nitrogen gas was $50 \mathrm{sccm}$ at atmospheric pressure. The etching rate of silicon carbide was determined by the decrease in weight and thickness of the substrate. The surface morphology was evaluated using the phase shift interference microscopy.

In this study, the specular silicon carbide surface obtained by this technique was utilized for the evaluation. The significant etching depth pattern was evaluated by observing the image of the bar-shaped light reflected at the mirror surface. The wafer distortion was macroscopically evaluated using the image of the bar-shaped light and the cross hatched patterns reflected at the specular surface obtained after the etching.

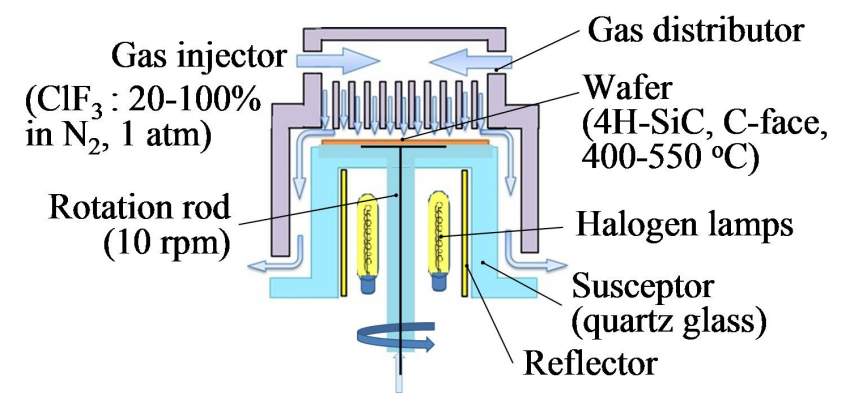

Fig. 1 Cross-sectional view of silicon carbide dry etcher.

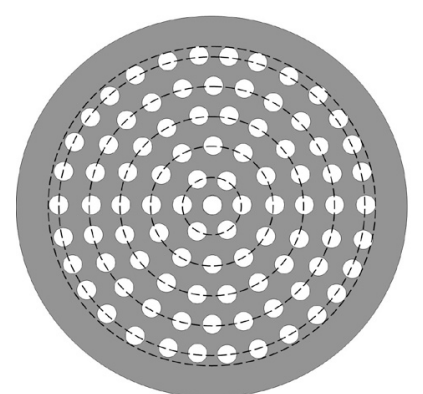

Fig. 2 Gas distributer (Dotted lines: concentric circles of arranged pin-holes).

\section{Results and Discussion}

The etching depth behavior was first evaluated. In order to verify the etching depth profile caused by the arrangement of the pin-hole of the gas distributor, predicted by the numerical calculation [3, 4], the wafer was significantly etched by the $100 \%$ chlorine trifluoride gas at the temperature slightly higher than $500{ }^{\circ} \mathrm{C}$ for 4 minutes. The average etching depth was about $15 \mu \mathrm{m}$. The wafer center was perfectly etched off to produce a hole.

The surface profile of the mirror polished wafer before etching was shown in Fig. 3 (a). This figure shows that the surface height was between $+1 \mathrm{~nm}$ and $-1 \mathrm{~nm}$. The etching depth profile was easily recognized by the visual inspection using the patterns of the reflected light, when the etched surface was specular. As shown in Fig. 3 (b), the etched surface was specular even after the deep etching. Fig. 3 (b) shows the bar-shaped light image reflected as the zig-zag pattern at the etched surface. This clearly shows the existence of macroscopic hills and valleys, as schematically shown in this figure. Additionally, the valleys were considered to exist near the positions of the pin-hole circles.

In order to recognize the relationship of the hills and valleys with the pin-hole position of the gas distributor, the dotted lines corresponding to the concentric circles on which the pin-holes were arranged as shown in Fig. 2, were drawn in Fig. 3 (b). There were the periodically arranged circle-shaped valleys, which agreed with the pin-hole circles. The pin hole design strongly influenced on the etching depth, when the surface was deeply etched. This results verified that the local etching depth profile could be formed on the single crystalline $4 \mathrm{H}$-silicon carbide, following the local chlorine trifluoride gas distribution. This result agreed with that for the polycrystalline $3 \mathrm{C}$-silicon carbide, while the $4 \mathrm{H}$-silicon carbide was finished to the mirror surface. In other words, the local etching profile can be adjusted by optimizing the local chlorine trifluoride gas supply, for performing the mirror etching. Fig. 3 (c) shows the surface profile measured at the point indicated by letter $\mathrm{x}$ in Fig. 3 (b). There was a wide terrace, although some etching pits existed. The surface showed a slope, consistently with Fig. 3 (b). As indicated using two parallel dotted lines in the bottom half of Fig. 3 (b), the surface height was within the range of $2 \mathrm{~nm}$. Thus, the surface microroughness was comparable to that before etching. 


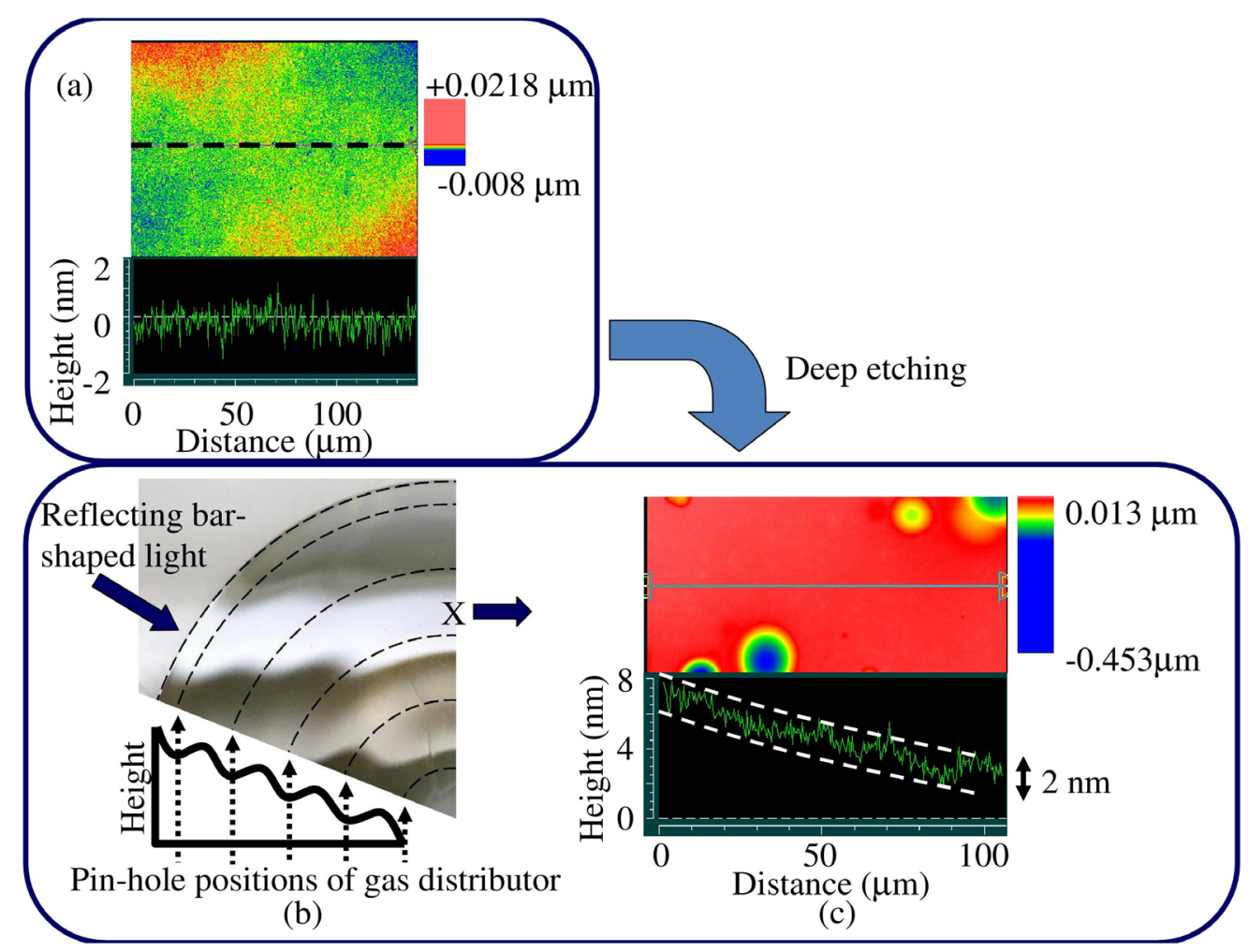

Fig. 3 4H-silicon carbide wafer surface (a) surface profile before etching, (b) wafer surface after significantly deep etching (reflecting the bar-shaped light) and (c) surface profile at point X in (b).

Next, the wafer stress, caused by the process in this study, was evaluated using the wafer distortion. The thin wafer, such as used in this study, tends to be distorted by the thermal and mechanical stresses. Additionally, the specular surface achieved by this technique enabled the visual inspection, using the periodically designed crossing lines reflected by the mirror finished $4 \mathrm{H}$-silicon carbide surface. The distortion of the cross hatched patterns observed over the wafer can show the situation of the wafer stress.

The silicon carbide wafer was etched sequentially four times, using the heat cycles between room temperature and $500{ }^{\circ} \mathrm{C}$. Fig. 4 shows the cross hatched patterns reflected at the wafer surface, (a) before etching, (b) after etching by $50 \%$ chlorine trifluoride gas at $500{ }^{\circ} \mathrm{C}$ for 5 minutes (1st etching), (c) after the additional etching by $50 \%$ chlorine trifluoride gas at $500{ }^{\circ} \mathrm{C}$ for 5 minutes (2nd etching), (d) after the additional etching by $100 \%$ chlorine trifluoride gas at $500{ }^{\circ} \mathrm{C}$ for 2 minutes (3rd etching), and (e) after further additional etching by $100 \%$ chlorine trifluoride gas at $500{ }^{\circ} \mathrm{C}$ for 4 minutes (4th etching). The numerical values written at top right position in these figures are the wafer thickness after the etching.

Fig. 4 (a) showed the straight lines over the mirror polished wafer. It might have slight slope due to the beveling near the edge. Figs. 4 (b)-(e) show that the etched wafer surfaces were specular and the obtained surface reflected the cross hatched patterns. The cross hatched patterns shown in Figs. 4 (b), (c) and (d) were entirely similar to that in Fig. 4 (a). The difference between Figs. 4 (c) and (d) was ignorable. Fig. 4 (e) showed that the reflected cross hatched patterns were very slightly bent, after the four times repetition of the heating up and down. Because the chemical etching generally leaves no stress at the etched surface, unlike the mechanical polishing, the wafer distortion could be small even for the very thin wafer, about $160 \mu \mathrm{m}$ thick. Conclusions

The 50-mm-diameter C-face single crystalline $4 \mathrm{H}$-silicon carbide wafer was etched using chlorine trifluoride gas at $500{ }^{\circ} \mathrm{C}$ for developing the silicon carbide wafer etching reactor. The etching depth profile corresponded to the circular-shaped array of the pin-hole arrangement of the gas distributor. Thus, the pin-hole arrangement is the key factor for locally adjusting the etching depth profile. The surface microroughness after the etching was similar to that before etching. The mirror etching of $\mathrm{C}$-face $4 \mathrm{H}$-silicon carbide wafer was possible. The wafer distortion was small, even when the wafer was very thin. 


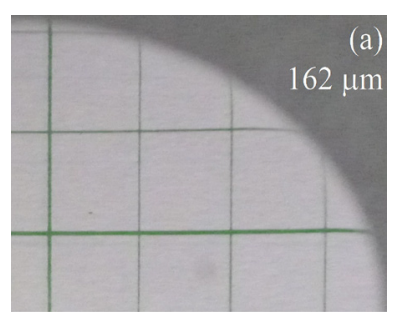

(a): Before etching

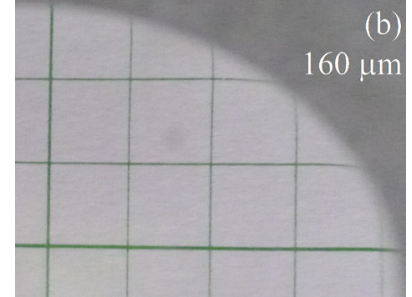

(b): (a) $+\mathrm{ClF}_{3} 50 \%, 5 \mathrm{~min}$

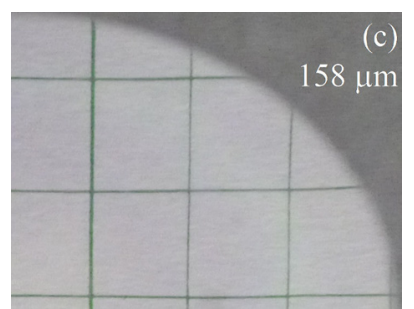

(c): (b) $+\mathrm{ClF}_{3} 50 \%, 5 \mathrm{~min}$

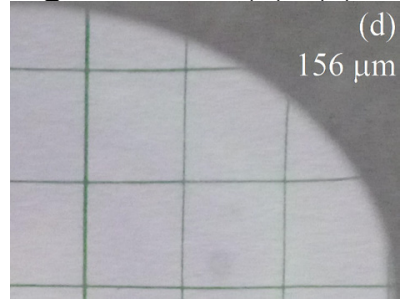

(d): (c) $+\mathrm{ClF}_{3} 100 \%, 2$ min

(e): (d) $+\mathrm{ClF}_{3} 100 \%, 4 \mathrm{~min}$

Fig. 4 Cross-hatched patterns reflected at the mirror surface of $\mathrm{C}$-face $4 \mathrm{H}$-silicon carbide wafer after the etching at $500{ }^{\circ} \mathrm{C}$.

\section{References}

[1] H. Habuka, K. Tanaka, Y. Katsumi, N. Takechi, K. Fukae, and T. Kato, J. Electrochem. Soc., 156 (2009) H971-H975.

[2] Y. Miura, Y. Katsumi, S. Oda, H. Habuka, Y. Fukai, K. Fukae, T. Kato, H. Okumura and K. Arai, Jpn. J. Appl. Phys. 46 (2007) 7875-7879.

[3] D. Yajima, Y. Fukumoto, H. Habuka and T. Kato, The 73th JSAP Autumn Meeting, 12p-H7-3 (2012).

[4] D. Yajima, H. Habuka and T. Kato, Mater. Sci. Forum, 778-780 (2014) 738-741.

[5] D. Yajima, K. Nakagomi, H. Habuka and T. Kato, Mater. Sci. Forum, 821-823 (2015) 553-556.

[6] K. Nakagomi, S. Okuyama, H. Habuka, Y. Takahashi and T. Kato, Materials Science Forum, 897 (2017) 383-386.

[7] S. Okuyama, K. Kurashima, H. Habuka, Y. Takahashi and T. Kato, ECS J. Solid State Sci. Technol., 6 (2017) P582-P585. 